Chapter 12

\title{
Where Is the PdV in the First Law of Black Hole Thermodynamics?
}

\author{
Brian P. Dolan
}

Additional information is available at the end of the chapter

http://dx.doi.org/10.5772/52455

\section{Introduction}

Ever since Hawking's discovery in 1974, [1-3], that black holes have a temperature associated to them, in the simplest case a temperature inversely proportional to their mass,

$$
T=\frac{\hbar}{8 \pi G M}
$$

(we use units in which $c=1$ ), the thermodynamics of black holes has been a fascinating area of research. Equation (1) immediately implies that a Schwarzschild black hole in isolation is unstable: it will radiate and in so doing loses energy hence the mass decreases, thus increasing the temperature causing it to radiate with more power leading to a runaway effect.

Hawking's result is fundamentally quantum mechanical in nature and came after a number of important developments in the classical thermodynamics of black holes. Penrose [4] realised that the mass of a rotating black hole can decrease, when rotational energy is extracted, and this was followed by the observation that the area never decreases in any classical process. Nevertheless there is still a minimum, irreducible, mass below which one cannot go classically $[5,6]$. This lead Bekenstein's to propose that an entropy should be associated with a black hole that is proportional to the area, $A$, of the event horizon, $[7,8]$. In natural units,

$$
S=\alpha \frac{A}{\hbar G}
$$

where $\alpha$ is an undetermined constant, presumed of order one, and $\hbar G$ is the Planck length squared. In the classical limit the temperature vanishes and the entropy diverges. 
The first law of black hole thermodynamics, in its simplest form, associates the internal energy of a black hole with the mass, $U(S)=M$, (more precisely the ADM mass, as defined with reference to the time-like Killing vector at infinity [9]) and reads

$$
d U=T d S
$$

In particular, for a Schwarzschild black hole, the event horizon radius is $r_{h}=2 G M$ and the event horizon area is

$$
A=4 \pi r_{h}^{2}=16 \pi G^{2} M^{2} \quad \Rightarrow \quad M=\frac{1}{4 G} \sqrt{\frac{A}{\pi}}
$$

Hence

$$
U=\frac{1}{4} \sqrt{\frac{\hbar S}{\pi \alpha G}}
$$

and

$$
T=\frac{\partial U}{\partial S}=\frac{1}{8} \sqrt{\frac{\hbar}{\pi \alpha G S}}=\frac{\hbar}{32 \pi \alpha G M} .
$$

Hawking's result (1) then fixes the constant of proportionality in (2) to be one quarter.

The black hole instability referred to above is reflected in the thermodynamic potentials by the fact that the heat capacity of a Schwarzschild black hole,

$$
C=T \frac{\partial S}{\partial T}=-\frac{\hbar G}{8 \pi T^{2}}<0
$$

is negative.

The first law generalises to electrically charged, rotating black holes as

$$
d U=T d S+\Omega d J+\Phi d Q
$$

where $J$ is the angular momentum of the black hole, $\Omega$ its angular velocity, and $Q$ the electric charge and the electrostatic potential (see e.g. [9]).

In contrast to elementary treatments of the first law of black hole thermodynamics it is noteworthy that (8) lacks the familiar $P d V$ term, but a little thought shows that it is by no means obvious how to define the volume of a black hole. For a Schwarzschild black hole the radial co-ordinate, $\mathrm{r}$, is time-like inside the event horizon, where $r<r_{h}$, so it would seem non-sensical to associate a volume $V=4 \pi \int_{0}^{r_{h}} r^{2} d r=\frac{4 \pi}{3} r_{h}^{3}$ with the black hole. In fact identifying any function of $r_{h}$ alone with a volume, $V\left(r_{h}\right)$, will lead to inconsistencies in a thermodynamic description since the area, and hence the entropy, is already a function of $r_{h}, S=\pi r_{h}^{2}$, so any volume $V\left(r_{h}\right)$ would be determined purely in terms of the entropy. The internal energy, $U(S, V)$, should be a function of two variables, so giving $V(S)$ uniquely 
as a specific function of $S$ is liable to lead to inconsistencies. We shall see below how this potential problem is avoided.

\section{Pressure and enthalpy}

From the point of view of Eintein's equations a pressure is associated with a cosmological constant. There is now very strong evidence that the cosmological constant in our Universe is positive [10, 11]. This poses a problem for the study of black hole thermodynamics for two reasons: firstly there is no asymptotic regime in de Sitter space which allows the unambiguous identification of the ADM mass of a black hole embedded in a space with a positive $\Lambda$; secondly positive $\Lambda$ corresponds to negative pressure, implying thermodynamic instability. The first problem is related to the fact that there are two event horizons for a de Sitter black hole, a black hole horizon and a cosmological horizon, and the radial co-ordinate is time-like for large enough values of $r$, outside the cosmological horizon. The second problem is not necessarily too serious as one can still glean some information from negative pressure systems which are thermodynamically unstable [12] (instability is not an insurmountable barrier to obtaining physical information from a thermodynamic system, after all, as described above, Hawking's formula (1) shows that black holes can have negative heat capacity but it is still a central formula in the understanding of black hole thermodynamics). In contrast for negative $\Lambda$ there is no cosmological horizon and the pressure is positive, the thermodynamics is perfectly well defined, so we shall restrict our considerations here to negative $\Lambda$ and identify the thermodynamic pressure $P=-\frac{\Lambda}{8 \pi G}$ with the fluid dynamical pressure appearing in Einstein's equations.

The notion that the cosmological constant should be thought of as a thermodynamic variable is not new, and its thermodynamic conjugate is often denoted $\Theta$ in the literature, [13-21], but $\Theta$ was not given a physical interpretation in these works.

It may seem a little surprising to elevate $\Lambda$ to the status of a thermodynamic variable. $\Lambda$ is usually thought of as a coupling constant in the Einstein action, on the same footing as Newton's constant, and it would seem bizarre to think of Newton's constant as a thermodynamic variable. However the nature of $\Lambda$ has long been mysterious [22] and we should keep an open mind as to its physical interpretation. Indeed in [23] it was argued that $\Lambda$ must be included in the pantheon of thermodynamic variables for consistency with the Smarr relation [24], which is essentially dimensional analysis applied to thermodynamic functions. Furthermore [23] suggested that, for a black hole embedded in anti-de Sitter (AdS) space-time, the black hole mass is more correctly interpreted as the enthalpy, $H$ beloved of chemists, rather than the more traditional internal energy,

$$
M=H(S, P)=U(S, V)+P V .
$$

The $P V$ term in this equation can be though of as the contribution to the mass-energy of the black hole due the negative energy density of the vacuum, $\epsilon=-P$, associated with a positive cosmological constant. If the black hole has volume $V$ then it contains energy $\epsilon V=-P V$ and so the total energy is $U=M-P V$.

This interpretation forces us to face up to the definition of the black hole volume. In [23] $V$ is defined as the volume relative to that of empty AdS space-time: the black hole volume is the volume excluded from empty AdS when the black hole is introduced. We shall refer to 
this as the 'geometric volume' below. Other suggestions for the volume of a black hole have been made in $[25,26]$

An alternative definition of the black-volume is that it is the thermodynamic conjugate of the pressure, under the Legendre transform (9),

$$
V:=\frac{\partial H}{\partial P}
$$

which we shall call the 'thermodynamic volume'.

With the definition of the thermodynamic volume (10) we are in a position to state the definitive version of the first law of black hole thermodynamics,

$$
d U=T d S+\Omega d J+\Phi d Q-P d V
$$

which follows from the Legendre transform of

$$
d M=d H=T d S+\Omega d J+\Phi d Q+V d P .
$$

Equation (12), in $\Theta d \Lambda$ notation, appeared in [27].

\section{Thermodynamic volume}

The suggested definition of the thermodynamic volume (10) must be tested for consistency. For example, for a non-rotating black hole in four-dimensional space-time, the line element is given, in Schwarzschild co-ordinates, by,

$$
d^{2} s=-f(r) d t^{2}+f^{-1}(r) d r^{2}+r^{2} d \Omega^{2},
$$

with

$$
f(r)=1-\frac{2 m}{r}-\frac{\Lambda}{3} r^{2}
$$

and $d \Omega^{2}=d \theta^{2}+\sin ^{2} \theta d \phi^{2}$ the solid angle area element. ${ }^{1}$ The event horizon is defined by $f\left(r_{h}\right)=0$,

$$
\frac{\Lambda}{3} r_{h}^{3}-r_{h}+2 m=0
$$

but we do not need to solve this equation explicitly in order to analyse (10). We already know that

$$
S=\pi r_{h}^{2}, \quad P=-\frac{\Lambda}{8 \pi}
$$

\footnotetext{
${ }^{1}$ From now on we set $G=\hbar=1$ to avoid cluttering formulae.
} 
and, for negative $\Lambda$, the ADM mass is $M=m$ [15], which, following the philosophy of [23], we identify with the enthalpy, $H(S, P)$. Solving (15) for $m$ immediately yields

$$
m=\frac{r_{h}}{2}\left(1-\frac{\Lambda}{3} r_{h}^{2}\right)
$$

from which $H(S, P)=M=m$, with (16), identifies the enthalpy as

$$
H(S, P)=\frac{1}{2}\left(\frac{S}{\pi}\right)^{\frac{1}{2}}\left(1+\frac{8 S P}{3}\right)
$$

The usual thermodynamic relations can now be used to determine the temperature and the volume,

$$
\begin{aligned}
& T=\left(\frac{\partial H}{\partial S}\right)_{P} \Rightarrow T=\frac{1}{4}\left(\frac{1}{\pi S}\right)^{\frac{1}{2}}(1+8 P S)=\frac{\left(1-\Lambda r_{h}^{2}\right)}{4 \pi r_{h}} \\
& V=\left(\frac{\partial H}{\partial P}\right)_{S} \Rightarrow V=\frac{4}{3} \frac{S^{\frac{3}{2}}}{\sqrt{\pi}}=\frac{4 \pi r_{h}^{3}}{3} .
\end{aligned}
$$

That the resulting thermodynamic volume (for a non-rotating black hole) is identical to the geometric volume is quite remarkable, but appears co-incidental as this equality no longer holds for rotating (Kerr-AdS) black holes, as we shall see. It does however hold for non-rotating black holes in all dimensions [28].

As mentioned in the introduction, equation (20) has a potential problem associated with it, in that it implies that the volume and the entropy cannot be considered to be independent thermodynamic variables, $S$ determines $V$ uniquely - they cannot be varied independently and so $V$ seems redundant. Indeed this may the reason why $V$ was never considered in the early literature on black hole thermodynamics. But this is an artifact of the non-rotating approximation, $V$ and $S$ can, and should, be considered to be independent variables for a rotating black hole.

The line element for a charged rotating black hole in 4-dimensional AdS space is [29]

$$
d s^{2}=-\frac{\Delta}{\rho^{2}}\left(d t-\frac{a \sin ^{2} \theta}{\Xi} d \phi\right)^{2}+\frac{\rho^{2}}{\Delta} d r^{2}+\frac{\rho^{2}}{\Delta_{\theta}} d \theta^{2}+\frac{\Delta_{\theta} \sin ^{2} \theta}{\rho^{2}}\left(a d t-\frac{r^{2}+a^{2}}{\Xi} d \phi\right)^{2}
$$

where

$$
\begin{aligned}
\Delta & =\frac{\left(r^{2}+a^{2}\right)\left(L^{2}+r^{2}\right)}{L^{2}}-2 m r+q^{2}, \quad \Delta_{\theta}=1-\frac{a^{2}}{L^{2}} \cos ^{2} \theta, \\
\rho^{2} & =r^{2}+a^{2} \cos ^{2} \theta, \quad \Xi=1-\frac{a^{2}}{L^{2}},
\end{aligned}
$$

and the cosmological constant is $\Lambda=-\frac{3}{L^{2}}=-8 \pi P$. 
The physical properties of this space-time are well known [15]. The metric parameters $m$ and $q$ are related to the ADM mass $M$ and the electric charge $Q$ by

$$
M=\frac{m}{\Xi^{2}}, \quad Q=\frac{q}{\Xi} .
$$

The event horizon, $r_{+}$, lies at the largest root of $\Delta(r)=0$, so, in terms of geometrical parameters,

$$
M=\frac{\left(r_{+}^{2}+a^{2}\right)\left(L^{2}+r_{+}^{2}\right)+q^{2} L^{2}}{2 r_{+} L^{2} \Xi^{2}}
$$

and the area of the event horizon is

$$
A=4 \pi \frac{r_{+}^{2}+a^{2}}{\Xi}
$$

giving

$$
S=\pi \frac{r_{+}^{2}+a^{2}}{\Xi} .
$$

The angular momentum is $J=a M$ and the relevant thermodynamic angular velocity is

$$
\Omega=\frac{a\left(L^{2}+r_{+}^{2}\right)}{L^{2}\left(r_{+}^{2}+a^{2}\right)}
$$

As explained in [27], $\Omega$ here is the difference between the asymptotic angular velocity and the angular velocity at the black hole outer horizon.

The electrostatic potential, again the difference between the potential at infinity and at the horizon, is

$$
\Phi=\frac{q r_{+}}{r_{+}^{2}+a^{2}}
$$

To determine the thermodynamic properties, $M$ must be expressed in terms of $S, J, Q$ and $P$ (or, equivalently, L). This was done in [27] and the result is

$$
H(S, P, J, Q):=\frac{1}{2} \sqrt{\frac{\left(S+\pi Q^{2}+\frac{8 P S^{2}}{3}\right)^{2}+4 \pi^{2}\left(1+\frac{8 P S}{3}\right) J^{2}}{\pi S}} .
$$

This generalises the Christodoulou-Ruffini formula [5, 6] for the mass of a rotating black hole in terms of its irreducible mass, $M_{i r r}$. (The irreducible mass for a black hole with entropy $S$ is the mass of a Schwarzschild black hole with the same entropy, $M_{i r r}^{2}=\frac{S}{4 \pi}$ ). 
The temperature follows from

$$
T=\left.\frac{\partial H}{\partial S}\right|_{J, Q, P}=\frac{1}{8 \pi H}\left[\left(1+\frac{\pi Q^{2}}{S}+\frac{8 P S}{3}\right)\left(1-\frac{\pi Q^{2}}{S}+8 P S\right)-4 \pi^{2}\left(\frac{J}{S}\right)^{2}\right],
$$

from which we immediately see that $T \geq 0$ requires

$$
J^{2} \leq \frac{S^{2}}{4 \pi^{2}}\left(1+\frac{\pi Q^{2}}{S}+\frac{8 P S}{3}\right)\left(1-\frac{\pi Q^{2}}{S}+8 P S\right)
$$

The maximum angular momentum,

$$
\left|J_{\max }\right|=\frac{S}{2 \pi} \sqrt{\left(1+\frac{\pi Q^{2}}{S}+\frac{8 P S}{3}\right)\left(1-\frac{\pi Q^{2}}{S}+8 P S\right)}
$$

is associated with an extremal black hole.

From (10) and (29) the thermodynamic volume is [30]

$$
V=\left.\frac{\partial H}{\partial P}\right|_{S, J, Q}=\frac{2}{3 \pi H}\left[S\left(S+\pi Q^{2}+\frac{8 P S^{2}}{3}\right)+2 \pi^{2} J^{2}\right]
$$

which is manifestly positive.

The angular velocity and the electric potential also follow from (29) via

$$
\Omega=\left.\frac{\partial H}{\partial J}\right|_{S, Q, P}=\frac{4 \pi^{2} J\left(1+\frac{8 P S}{3}\right)}{2 H \sqrt{\pi S}}
$$

and

$$
\Phi=\left.\frac{\partial H}{\partial Q}\right|_{S, J, P}=\frac{2 \pi Q\left(S+\pi Q^{2}+\frac{8 P S^{2}}{3}\right)}{2 H \sqrt{\pi S}} .
$$

The Smarr relation follows from (29), (30), (33), (34) and (35), namely

$$
\frac{H}{2}+P V-S T-J \Omega-\frac{Q \Phi}{2}=0
$$

from which it is clear that the $P V$-term must be included for consistency, as pointed out in [23]. 
It is clear from (33) that, in general, $V$ is a function of all the four independent thermodynamical variables, $S, P, J$ and $Q$, but for the limiting case $J=0$,

$$
V=\frac{4}{3} \frac{S^{\frac{3}{2}}}{\sqrt{\pi}}
$$

is determined purely in terms of $S$ alone, independent of both $P$ and $Q$. Thus, as explained in the introduction, $V$ and $S$ cannot be viewed as thermodynamically independent variables as $J \rightarrow 0$, rendering the description in terms of the thermodynamic potential $U(S, J)$ impossible in this limit.

Expressing the thermodynamic volume (33) in terms of geometrical variables one gets [30]

$$
V=\frac{2 \pi}{3}\left\{\frac{\left(r_{+}^{2}+a^{2}\right)\left(2 r_{+}^{2} L^{2}+a^{2} L^{2}-r_{+}^{2} a^{2}\right)+L^{2} q^{2} a^{2}}{L^{2} \Xi^{2} r_{+}}\right\} .
$$

Given that the area of the event horizon is

$$
A=4 \pi \frac{r_{+}^{2}+a^{2}}{\Xi}
$$

then, if we define a naïve volume

$$
V_{0}:=\frac{r_{+} A}{3}=\frac{4 \pi}{3} \frac{r_{+}\left(r_{+}^{2}+a^{2}\right)}{\Xi},
$$

equations (24) and (38) give

$$
V=V_{0}+\frac{4 \pi a^{2} M}{3}=V_{0}+\frac{4 \pi}{3} \frac{J^{2}}{M}
$$

a formula first derived in [31]. As pointed out in that reference, equation (40) implies that the surface to volume ratio of a black hole is always less than that of a sphere with radius $r_{+}$in Euclidean geometry. This is the opposite of our usual intuition that a sphere has the smallest surface to volume ratio of any closed surface - the isoperimetric inequality of Euclidean geometry. Thus the surface to volume ratio of a black hole satisfies the reverse of the usual isoperimetric inequality (a similar result holds in higher dimensions [31]). At least this seems to be the case if quantum gravity effects are not taken into account. In one case where quantum gravity corrections can be calculated using the techniques in [32], the three-dimensional Bañados-Zanelli-Teitelboim (BTZ) black hole [33], they tend to reduce the black hole volume [28] so it seems possible that quantum gravity effects may affect the reverse isoperimetric inequality. 


\section{The First Law}

To examine the consequences of the $P d V$ term in the first law we need to perform a Legendre transform on the enthalpy to obtain the internal energy $U(S, V, J, Q)$ from $U=H-P V$. We first write the enthalpy (29) in the form

$$
H=\sqrt{a+b P+c P^{2}},
$$

where

$$
\begin{aligned}
a & :=\frac{\pi}{S}\left\{\frac{1}{4}\left(\frac{S}{\pi}+Q^{2}\right)^{2}+J^{2}\right\} \\
b & :=\frac{4 \pi}{3}\left\{\frac{S}{\pi}\left(\frac{S}{\pi}+Q^{2}\right)+2 J^{2}\right\} \\
c & :=\left(\frac{4 \pi}{3}\right)^{2}\left(\frac{S}{\pi}\right)^{3} .
\end{aligned}
$$

Note that the discriminant,

$$
b^{2}-4 a c=\frac{64 \pi^{2}}{9} J^{2}\left(J^{2}+\frac{S Q^{2}}{\pi}\right)
$$

is positive.

Now

$$
V=\left.\frac{\partial H}{\partial P}\right|_{S, J, Q}=\frac{b+2 c P}{2 H} \quad \Rightarrow \quad P=\frac{2 H V-b}{2 c} .
$$

This allows us to re-express $H$ as a function of $V$,

$$
H=\frac{1}{2} \sqrt{\frac{b^{2}-4 a c}{V^{2}-c}} .
$$

We can immediately conclude that

$$
V^{2} \geq c=\left(\frac{4 \pi}{3}\right)^{2}\left(\frac{S}{\pi}\right)^{3}
$$

with equality only when

$$
b^{2}-4 a c=\frac{64 \pi^{2}}{9} J^{2}\left(J^{2}+\frac{S Q^{2}}{\pi}\right)=0,
$$


i.e. when $J=0$.

It is now straightforward to determine

$$
U=H-P V=H-\left(\frac{H V^{2}}{c}-\frac{b V}{2 c}\right)=\frac{b V}{2 c}-\frac{\sqrt{\left(V^{2}-c\right)\left(b^{2}-4 a c\right)}}{2 c},
$$

which immediately gives

$$
\begin{aligned}
& U(S, V, J, Q)=\left(\frac{\pi}{S}\right)^{3} {\left[\left(\frac{3 V}{4 \pi}\right)\left\{\left(\frac{S}{2 \pi}\right)\left(\frac{S}{\pi}+Q^{2}\right)+J^{2}\right\}\right.} \\
&\left.-|J|\left\{\left(\frac{3 V}{4 \pi}\right)^{2}-\left(\frac{S}{\pi}\right)^{3}\right\}^{\frac{1}{2}}\left(\frac{S Q^{2}}{\pi}+J^{2}\right)^{\frac{1}{2}}\right] .
\end{aligned}
$$

Note the subtlety in the $J \rightarrow 0$ limit, (50) is not differentiable at $J=0$ unless

$$
\left(\frac{3 V}{4 \pi}\right)^{2}=\left(\frac{S}{\pi}\right)^{3}
$$

there.

Equation (50) can now be used to study the efficiency of a Penrose process. If a black hole has initial mass $M_{i}$, with internal energy $U_{i}$, and is taken through a quasi-static series of thermodynamic steps to a state with final internal energy $U_{f}$, then energy can be extracted if $U_{f}<U_{i}$. This is the thermodynamic description of a Penrose process [4] and the efficiency is

$$
\eta=\frac{U_{i}-U_{f}}{M_{i}}
$$

We can determine the maximum efficiency for a process at constant $P$ by first expressing $U$ in (50) in terms of $S, P, J$ and $Q$ :

$$
U=\frac{\left(S+\pi Q^{2}\right)\left(S+\pi Q^{2}+\frac{8 P S^{2}}{3}\right)+4 \pi^{2}\left(1+\frac{4 P S}{3}\right) J^{2}}{2 \sqrt{\pi S\left[\left(S+\pi Q^{2}+\frac{8 P S^{2}}{3}\right)^{2}+4 \pi^{2}\left(1+\frac{8 P S}{3}\right) J^{2}\right]}},
$$

which is manifestly positive.

For simplicity consider first the $Q=0$ case, for which

$$
d U=T d S+\Omega d J-P d V
$$


The work extracted at any infinitesimal step is

$$
d W=-d U=-T d S-\Omega d J+P d V
$$

and, since $d S \geq 0$, this is maximised in an isentropic process $d S=0$. Now with $Q=0$ and $S$ and $P$ held constant, the internal energy in equation (53) can be thought of as a function of $J$ only, $U(J)$. The greatest efficiency is then obtained by starting with an extremal black hole and reducing the angular momentum from $J_{\max }$ to zero, it is given by

$$
\eta_{\text {ext }}=\frac{U\left(J_{\max }\right)-U(0)}{H\left(J_{\max }\right)}
$$

where $H\left(J_{\max }\right)=M_{\text {ext }}$ is the initial extremal mass. One finds

$$
\eta_{\text {ext }}=\frac{1+2 P S}{1+4 P S}-\frac{1}{\sqrt{2+8 P S}} \frac{3}{(3+8 P S)} .
$$

In asymptotically flat space, $\Lambda=0$, we set $P=0$ in $\eta_{\text {ext }}$ and obtain the famous result [9]

$$
\eta_{e x t}=1-\frac{1}{\sqrt{2}} \approx 0.2929
$$

More generally, $\eta_{\text {ext }}$ is a maximum for $S P=1.837 \ldots$ (obtained by solving a quartic equation) and attains there the value $0.5184 \ldots$. Thus turning on a negative cosmological constant increases the efficiency of a Penrose process, as first observed in [30].

What is happening here is that, as $|J|$ decreases (giving a positive contribution to $d W$ ) the volume decreases, which actually tends to decrease the work done because of the $P d V$ term in (55). But when $P>0$, the extremal value $\left|J_{\max }\right|$ in (32) is increased, which more than compensates, and overall $\eta_{\text {ext }}$ is increased.

For a charged black hole the internal energy is a function of $J$ and $Q$ for an isobaric isentropic process, $U(J, Q)$. The requirement $J_{\max }^{2} \geq 0$ in (32) imposes the constraint

$$
Q^{2} \leq Q_{\max }^{2}=\left(\frac{S}{\pi}\right)(1+8 P S)
$$

on the charge. The greatest efficiency is achieved starting from an extremal black hole with $Q^{2}=Q_{\text {max }}^{2}$ and reducing both $J$ and $Q$ to zero in the final state,

$$
\eta_{e x t}=\frac{U\left(J_{\max }, Q_{\max }\right)-J(0,0)}{H\left(J_{\max }, Q_{\max }\right)}=\frac{3}{2}\left(\frac{1+8 P S}{3+16 P S}\right)
$$

with $H\left(J_{\max }, Q_{\max }\right)$ the initial extremal mass, $M_{\text {ext }}$. For large $S$ efficiencies of up to $75 \%$ are possible [30], which should be compared to $50 \%$ in the $\Lambda=0$ case, [9]. 


\section{Critical behaviour}

With knowledge of both $H$ and $U$ general questions concerning the heat capacity of black holes can be addressed. The heat capacity at constant volume, $C_{V}=T /\left(\frac{\partial T}{\partial S}\right)_{V, J, Q^{\prime}}$ tends to zero when $J=0$, though $C_{V}$ can be non-zero for $J \neq 0$ it does not diverge. For comparison the heat capacity at constant pressure, $C_{P}=T /\left(\frac{\partial T}{\partial S}\right)_{P, J, Q^{\prime}} C_{P}$ vanishes when $T=0$ and diverges when $\frac{\partial T}{\partial S}=0$.

A full stability analysis was given in [27] and there are both local and global phase transitions. Local stability can be explored visually, by plotting thermodynamic functions, or analytically, examining the curvature of the derivatives of thermodynamics functions.

\section{1. $Q=0$}

Let us first focus on the $Q=0$ case. The blue (lower) curve in the figure below shows the locus of points where $C_{P}$ diverges in the $J-S$ plane, it is given by setting the denominator of $C_{P}$,

$$
144(\pi J P)^{4}(9+32 S P)+24(\pi P J)^{2}(P S)^{2}(3+16 S P)(3+8 S P)^{2}-(P S)^{4}(1-8 S P)(3+8 S P)^{3}
$$

to zero. The red (upper) curve is the $T=0$ locus, all points above and left of this curve are unphysical as $T<0$ in this region.

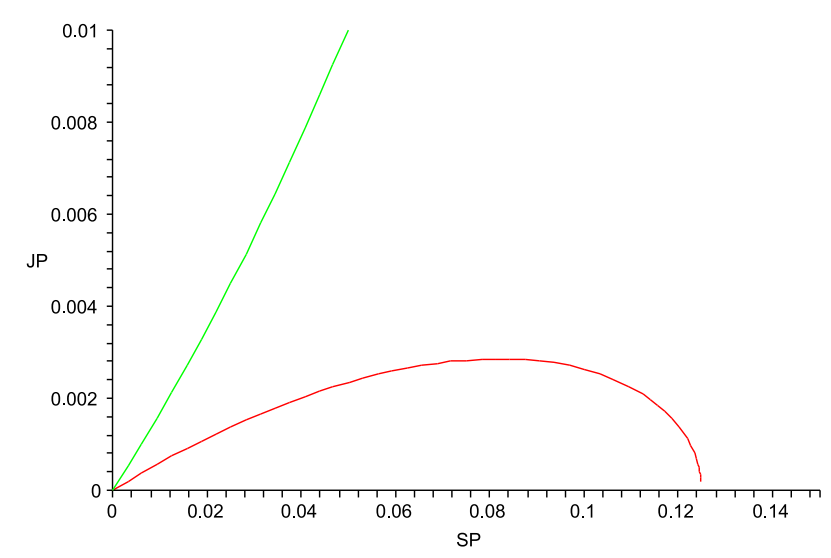

Figure 1. $T=0$ and $C_{P} \rightarrow \infty$ curves in $J-S$ plane.

There is also a global phase transition, not shown in the figure, when the free energy of pure AdS is lower than that of a black hole in asymptotically AdS space-time, the famous Hawking-Page phase transition [34]. We shall focus on the second order local phase transition here and examine its critical properties.

In general, at fixed $P$ and $J$, there are two values of $S$ at which $C_{P}$ diverges, and there is a critical point where these two values coalesce into one, the maximum of the lower curve in figure 1. This critical point was first identified in [27]. On purely dimensional grounds $P C_{P}$ 
can be expressed as a function of PS and PJ and the critical point can be found analytically, by solving a cubic equation, but the explicit form is not very illuminating. Numerically it lies at

$$
(P S)_{\text {crit }} \approx 0.08204, \quad(P J)_{\text {crit }} \approx 0.002857 .
$$

The critical temperature is obtained from (30), with $Q=0$,

$$
\left(\frac{T}{\sqrt{P}}\right)_{c r i t} \approx 0.7811
$$

and the critical volume likewise from (33)

$$
\left(V P^{3 / 2}\right)_{\text {crit }} \approx 0.01768
$$

(the authors of [27] fix $P=\frac{3}{8 \pi} \approx 0.1194$, corresponding to $L=1$, and find a critical value of $J$ at $J_{\mathcal{C}} \approx 0.0236$ ).

The equation of state cannot be obtained analytically, but its properties near the critical point can be explored by a series expansion and critical exponents extracted. Define the reduced temperature and volume as

$$
t=\frac{T-T_{c}}{T_{c}} \quad v=\frac{V-V_{c}}{V_{c}} .
$$

It is convenient to expand around the critical point using

$$
p:=16 \pi\left(P J-(P J)_{\text {crit }}\right)
$$

and

$$
q:=8\left(P S-(P S)_{\text {crit }}\right)
$$

Expanding the temperature (30) around the critical point, with $Q$ set to zero, gives

$$
t=2.881 p+2.201 p q+0.3436 q^{3}+o\left(p^{2}, p q^{2}, q^{4}\right)
$$

while similar expansion of the thermodynamic volume (33) yields

$$
v=-10.44 p+2.284 q+o\left(p^{2}, p q, q^{2}\right)
$$

For a given fixed $J>0, p$ is the deviation from critical pressure in units of $1 /(16 \pi J)$, but one must be aware that this interpretation precludes taking the $J \rightarrow 0$ limit in this formulation. Bearing this in mind, (68) and (69) give the $J>0, Q=0$ equation of state parametrically in terms of $q$. Eliminating $q$ one arrives at

$$
p=0.3472 t-0.1161 t v-0.02883 v^{3}+o\left(t^{2}, t v^{2}, v^{4}\right) .
$$


The critical exponent $\alpha$ is defined by

$$
C_{V} \propto t^{-\alpha}
$$

and, since as already stated, $C_{V}$ does not diverge at $t=0, \alpha=0$. To see this explicitly note that $C_{V}=T /\left.\frac{\partial T}{\partial S}\right|_{V}$ and

$$
\left.\frac{\partial T}{\partial S}\right|_{V}=\left.\frac{\partial T}{\partial S}\right|_{P}+\left.\left.\frac{\partial T}{\partial P}\right|_{S} \frac{\partial P}{\partial S}\right|_{V}=T_{C}\left(\left.\frac{\partial t}{\partial S}\right|_{P}+\left.\left.\frac{\partial t}{\partial P}\right|_{S} \frac{\partial P}{\partial S}\right|_{V}\right)
$$

Now, near the critical point, (68) gives

$$
\begin{aligned}
& \left.\frac{\partial t}{\partial S}\right|_{P}=\left.8 P \frac{\partial t}{\partial q}\right|_{p}=o\left(p, q^{2}\right), \\
& \left.\frac{\partial t}{\partial P}\right|_{S}=\left.8 S \frac{\partial t}{\partial q}\right|_{p}+\left.16 \pi J \frac{\partial t}{\partial p}\right|_{q}=2.881(16 \pi J)+o(p, q),
\end{aligned}
$$

while (69) implies $d p=0.2188 d q$ for constant $v$, from which is follows that $\left.\frac{\partial P}{\partial S}\right|_{V}$ is non-zero at the critical point, hence $\left.\frac{\partial T}{\partial S}\right|_{V}$ does not vanish at the critical point and so $\alpha=0$.

The exponent $\beta$ is defined by

$$
v_{>}-v_{<}=|t|^{\beta}
$$

where $v_{>}$is the greater volume and $v_{<}$the lesser volume across the phase transition, at constant pressure, when $t<0(v<$ is negative, since $v=0$ at the critical point).

Keeping $p$ and $t$ constant in (70) implies that

$$
p \int_{v_{<}}^{v_{>}} d v=0.3742 t \int_{v_{<}}^{v_{>}} d v-\int_{v_{<}}^{v_{>}}\left(0.1161 t v+0.02883 v^{3}\right) d v
$$

Allowing for the area of the rectangle in figure 3, namely $0.3742|t|\left(v_{>}-v_{<}\right)$, Maxwell's equal area law then requires

$$
\int_{v_{<}}^{v_{>}}\left(0.1161 t v+0.02883 v^{3}\right) d v=0 \quad \Rightarrow \quad|t| \propto\left(v_{>}^{2}+v_{<}^{2}\right) .
$$

It is clear from the figure that $v_{>}-v_{<} \gg v_{>}+v_{<}$so

$$
\left(v_{>}^{2}+v_{<}^{2}\right)=\frac{1}{2}\left(\left(v_{>}-v_{<}\right)^{2}+\left(v_{>}+v_{<}\right)^{2}\right) \approx \frac{1}{2}\left(v_{>}-v_{<}\right)^{2}
$$

giving 


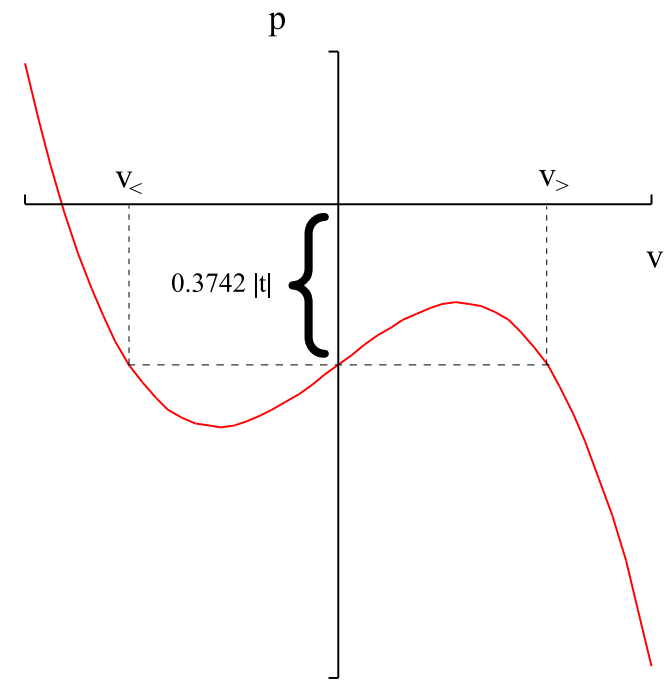

Figure 2. Construction associated with Maxwell's equal area law.

$$
|t| \propto\left(v_{>}-v_{<}\right)^{2}
$$

and $\beta=\frac{1}{2}$.

The critical exponent $\gamma$ is related to the isothermal compressibility,

$$
\kappa_{T}=-\frac{1}{V}\left(\frac{\partial V}{\partial P}\right)_{T, J}=-\frac{1}{V}\left(\frac{\partial V}{\partial P}\right)_{S, J}-\left(\frac{\partial V}{\partial S}\right)_{P, J} \frac{\left(\frac{\partial T}{\partial P}\right)_{S, J}}{\left(\frac{\partial T}{\partial S}\right)_{P, J}}
$$

which diverges along the same curve as $C_{P}$ does (the adiabatic compression, $\kappa_{S}=$ $-\frac{1}{V}\left(\frac{\partial V}{\partial P}\right)_{S, J^{\prime}}$ is everywhere finite - see equation (92)). $\gamma$ gives the divergence of the isothermal compressibility near the critical point,

$$
\kappa_{T} \propto t^{-\gamma}
$$

$\gamma$ can be found by expanding the denominator of $C_{P}$ in (61) around the critical point, but a quicker method, since we have the equation of state, is to differentiate (70) with respect to $v$, keeping $t$ constant, giving

$$
\left.\frac{\partial p}{\partial v}\right|_{t} \propto-t
$$


hence

$$
\kappa_{T} \propto-\left.\frac{\partial v}{\partial p}\right|_{t} \propto \frac{1}{t}
$$

and $\gamma=1$.

Lastly setting $t=0$ in (70) we see that

$$
|p| \propto|v|^{\delta}
$$

with $\delta=3$, again the mean field result.

To summarise, the critical exponents are

$$
\alpha=0, \quad \beta=\frac{1}{2}, \quad \gamma=1, \quad \delta=3
$$

These are the same critical exponents as the Van der Waals fluid and, more importantly, are mean field exponents. The same critical exponents have been found using a virial expansion in [50].

It was first pointed in $[35,36]$ that a non-rotating, charged black hole has a critical point of the same nature as that of of a Van der Waals fluid, and the critical exponents for the black hole phase transition in this case were calculated in [37] and verified to be mean field exponents, which are indeed the those of a Van der Waals fluid. A similarity between the neutral rotating black hole and the Van der Waals phase transition was first pointed out in [27] and further explored in [30].

The critical point can be visualised by plotting the Gibbs free energy

$$
G(T, P, J)=H(S, P, J)-T S,
$$

for $J=1$ and $Q=0$, as a function of $P$ and $T$ as in figure 3. We see the "swallow-tail catastrophe" that is typical of the Van der Waals phase transition [38].

This structure is a straightforward consequence of Landau theory, [39]. Near the critical point the Landau free energy is

$$
L(T, P, v)=G(T, P)+A\left\{(p-B t) v+C t v^{2}+D v^{4}\right\}+\ldots,
$$

where $G(T, P)$ is the Gibbs free energy and $A, B, C$ and $D$ are positive constants (for simplicity the constant $J$ is not made explicit). As stressed in [40] $L$ is not strictly speaking a thermodynamic function as it depends on three variables, $p, t$ and $v$ instead of two: $v$ is to be determined in terms of $p$ and $t$ by extremising $L$ to obtain the equation of state. 


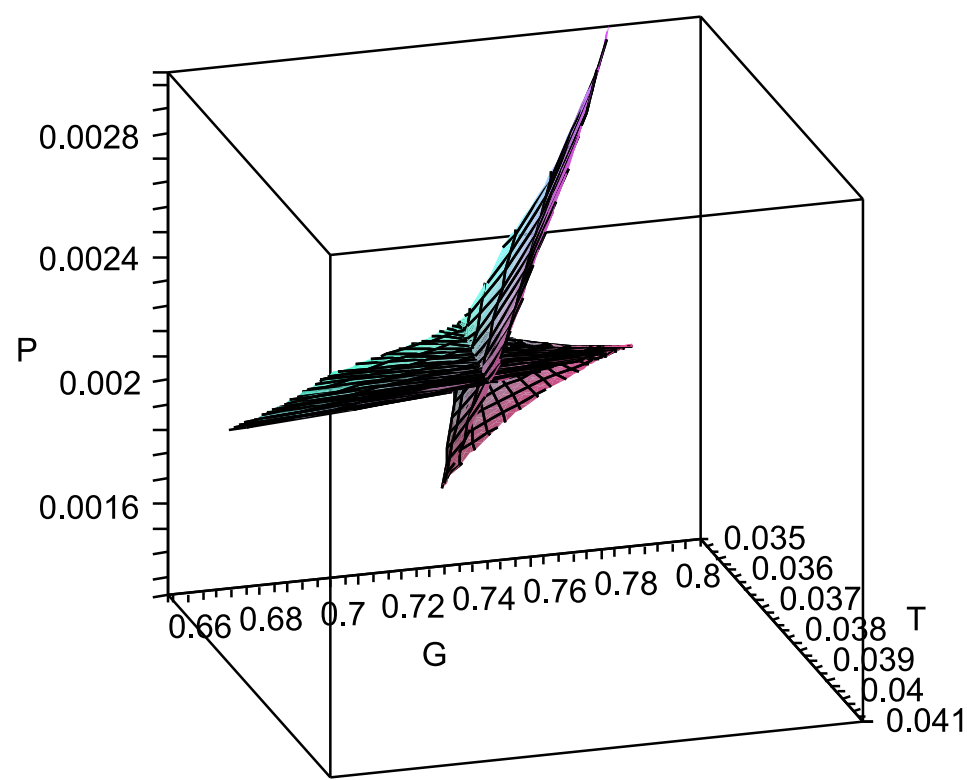

Figure 3. Gibbs free energy as a function of pressure and temperature, at fixed angular momentum.

For notational convenience equation (87) can be written, for fixed $p$ and $t$, as

$$
L=a+b v+c v^{2}+v^{4}
$$

where $a, b$ and $c$ need not be positive and $L \rightarrow \frac{1}{A D} L$ has been rescaled by a trivial positive constant. We are to think of $b$ and $c$ are control parameters that can be varied by varying $p$ and $t$.

Extremising (88) with respect to $v$ determines the value of $v$ in terms of $b$ and $c$ through

$$
b=-2 c v-4 v^{3} .
$$

Using this in $L$ leads to

$$
L=a-c v^{2}-3 v^{4} .
$$

Equations (89) and (90) together give $L(a, b, c)$ implicitly: a parametric plot of $L(b, c)$, for any fixed $a$, reveals a characteristic "swallow-tail catastrophe" structure. With hindsight the swallow-tail structure is clear: in the $A-D-E$ classification of critical points of functions, [41], (88) has three control parameters and is derived from type $A_{4}$ in Arnold's classification. 


\section{2. $Q \neq 0$}

The above structure was first found in AdS black hole thermodynamics in the charged $J=0$ case $[35,36]$, where the equation of state can be found exactly and the critical exponents can be determined [37]. When both $J$ and $Q$ are non-zero an analytic analysis is much more difficult, for example finding the zero locus of the denominator of $C_{P}$ requires solving a quintic equation. However numerical studies show that for a charged rotating black hole, as long as the charge is below the extremal value, the picture is qualitatively the same: the critical exponents are the same, the Landau free energy is still related to type $A_{4}$ and the Gibbs free energy still takes on a characteristic swallow-tail shape. For fixed values of $J$ and $Q$, not both zero, all that changes is the numerical value of the co-efficients in equations (68), (69) and (70) or, equivalently the numerical values of the constants $A, B, C$ and $D$ in (87). As long as none of these constants actually changes sign the nature of the critical point does not change and the critical exponents are the same.

\section{Compressibility and the speed of sound}

In the previous section, the nature of the singularity in the isothermal compressibility near the critical point was discussed, but the adiabatic compressibility

$$
\kappa_{S}=-\frac{1}{V}\left(\frac{\partial V}{\partial P}\right)_{T, J, Q}
$$

is also of interest, and this was studied in [42] on which most of this section is based. From (10) one finds, setting $Q=0$ for simplicity, that

$$
\kappa_{S}=\frac{36(2 \pi J)^{4} S}{(3+8 P S)\left\{(3+8 P S) S^{2}+(2 \pi J)^{2}\right\}\left\{2(3+8 P S) S^{2}+3(2 \pi J)^{2}\right\}} .
$$

This is finite at the critical point, indeed it never diverges for any finite values of $S, P$ and $J$, and it vanishes as $J \rightarrow 0$ : non-rotating black holes are completely incompressible. Black holes are maximally compressible in the extremal case $T=0$, when $J=J_{\max }$ in (32),

$$
\left.\kappa_{S}\right|_{T=0}=\frac{2 S(1+8 P S)^{2}}{(3+8 P S)^{2}(1+4 P S)} .
$$

A speed of sound, $c_{S}$, can also be associated with the black hole, in the usual thermodynamic sense that

$$
c_{S}^{-2}=\left.\frac{\partial \rho}{\partial P}\right|_{S, J}=1+\rho \kappa_{S}=1+\frac{9(2 \pi J)^{4}}{\left\{2(3+8 P S) S^{2}+3(2 \pi J)^{2}\right\}^{2}},
$$


where $\rho=\frac{M}{V}$ is the density. $c_{S}$ is unity for incompressible non-rotating black holes and is lowest for extremal black holes in which case

$$
\left.c_{S}^{-2}\right|_{T=0}=1+\left(\frac{1+8 P S}{3+8 P S}\right)^{2} .
$$

giving $c_{S}^{2}=0.9$ (in units with $c=1$ ) when $P=0$. In the limiting case $P S \rightarrow \infty, c_{S}^{2}$ achieves a minimum value of $1 / 2$.

These results show that the equation of state is very stiff for adiabatic variations of non-rotating black holes and gets softer as $J$ increases. For comparison, the adiabatic compressibility of a degenerate gas of $N$ relativistic neutrons in a volume $V$ at zero temperature follows from the degeneracy pressure

$$
P_{\text {deg }}=\left(3 \pi^{2}\right)^{\frac{1}{3}} \frac{c \hbar}{4}\left(\frac{V}{N}\right)^{-\frac{4}{3}} \Rightarrow \kappa_{S}=\frac{3}{4 P_{d e g}} .
$$

For a neutron star $\frac{N}{V} \approx 10^{45} \mathrm{~m}^{-3}$ and $\kappa_{S} \approx 10^{-34} \mathrm{~kg}^{-1} \mathrm{~ms}^{2}$. With zero cosmological constant the black hole adiabatic compressibility at zero temperature is given by (93) with $P=0$,

$$
\left.\kappa_{S}\right|_{T=P=0}=\frac{2 S}{9}=\frac{4 \pi M^{2} G^{3}}{9 c^{8}}
$$

where the relevant factors of $c$ and $G$ are included, and the entropy has been set to the extremum value of $2 \pi M$. Putting in the numbers

$$
\left.\kappa_{S}\right|_{T=0}=2.6 \times 10^{-38}\left(\frac{M}{M_{\odot}}\right)^{2} k g^{-1} m^{2},
$$

which is four orders of magnitude less than that of a solar-mass neutron star. We conclude that the zero temperature black hole equation of state, although "softer" than that of a non-rotating black hole, is still very much stiffer than that of a neutron star.

The "softest" compressibility for a neutral black hole however is the isothermal compressibility: for an extremal black hole

$$
\left.\kappa_{T}\right|_{T=0}=\frac{2 S\left(11+80 P S+128(P S)^{2}\right)}{(1+4 P S)\left(3+48 P S+128(P S)^{2}\right)} \underset{P \rightarrow 0}{\longrightarrow} \frac{22 S}{3},
$$

some 33 times larger than $\left.\kappa_{S}\right|_{T=P=0}$ in (97), but still much larger than degenerate matter in a solar-mass neutron star. 


\section{Open questions}

The obvious open question arising from the ideas presented here is: what about $\Lambda>0$ ?

The analysis of critical behaviour in $\S 5$ is only valid for $\Lambda<0$, this critical point lies deep in the region $P>0$ and does not appear to be of any relevance to astrophysical situations. It is certainly of interest in the AdS-CFT correspondence [43] but the particular analysis of $\S 5$, being in $1+3$-dimensions could only be relevant to $2+1$-dimensional conformal field theory, which is of course of interest in its own right [44]. Of course one could perform a similar analysis for 4+1-dimensional, or yet higher dimensional black holes, to try and gain insight into higher dimensional conformal field theory, and indeed this seems to have been the motivation in $[31,35,36]$, but these ideas are not the focus of this volume and will not be pursued here.

The thermodynamics of black holes in de Sitter space-time is a notoriously difficult problem $[17,20,45-48]$ as there are two event horizons and no "asymptotically de Sitter" region inside the cosmological horizon. Even with no black hole, a naïve interpretation of the cosmological horizon implies that the transition from $\Lambda=0$ to any infinitesimally small $\Lambda>0$ appears to involve a discontinuous jump from zero to infinite entropy, at least if one associates the usual Hawking-Bekenstein entropy with the cosmological horizon when $\Lambda>0$.

Nevertheless it is argued in [45] that a consistent strategy is to fix the relevant components of the metric at the cosmological horizon, rather than at spacial infinity as would be done in asymptotically flat or AdS space-time. When that is done the same expression for the ADM mass (24) is obtained, but with $L^{2} \rightarrow-L^{2}$, so $\Xi>1$ while the angular momentum is still given by $J=a M$. In this picture, all of the formulae in $\S 3$ are applicable for positive $\Lambda$ and negative $P$, provided $P$ is not too negative. If $\Lambda$ is too large the black hole horizon and the cosmological horizon coincide and demanding that this does not happen puts a lower bound on $P$, for any fixed $S, J$ and $Q$ : with $Q=0$, for example, this requirement constrains $P$ to

$$
P>\frac{\sqrt{S^{2}+12 \pi J^{2}}-2 S}{8 S} .
$$

Provided $P$ lies above this lower bound we can analytically continue (29) to negative $P$, with the understanding that $S$ is the entropy of the black hole event horizon only and does not include any contribution from the cosmological horizon.

Of course $P<0$ is thermodynamically unstable, but it can be argued, in some circumstances at least, that positive pressures can be analytically continued to negative pressures [12, 39], and in a cosmological context there can now be little doubt that $P<0$. Adopting the strategy of [45] the maximal efficiency of a rotating black hole in de Sitter space will be less than in the $\Lambda=0$ case, based on simply changing the sign of $\Lambda$ in $\S 4$, and the zero charge efficiency vanishes when the black hole horizon and the cosmological horizon coincide at $P S=-\frac{1}{8}$. Any such deviation from the $\Lambda=0$ case will however be completely negligible for astrophysical black holes around one solar mass and the observed value of $\Lambda$, but it could be more significant during periods of inflation when $\Lambda$ was larger.

It has been suggested that primordial black-holes may have formed in the early Universe [49] and, if this is the case and if they formed in sufficient numbers at any stage, then one should model the primordial gas as containing a distribution of highly incompressible black holes, 
like beads in a gas. These would certainly be expected to affect the overall compressibility of the gas as well as the speed of sound through the gas. In a radiation dominated Universe, ignoring the matter density, the speed of sound in the photon gas would be given by

$$
c_{\gamma}^{-2}=\left.\frac{\partial \epsilon}{\partial P}\right|_{S}=3 c^{-2},
$$

where $\epsilon$ is the energy density (essentially since the equation of state is $P=\frac{1}{3} \epsilon$ ) so $c_{\gamma}=0.577 c$. Since the speed of sound associated with the embedded black hole "beads" is $c_{S} \geq \sqrt{0.9} c=$ $0.9487 c$ the presence of a significant density of primordial black holes would expected to affect speed of sound in the photon gas and thus affect the dynamics.

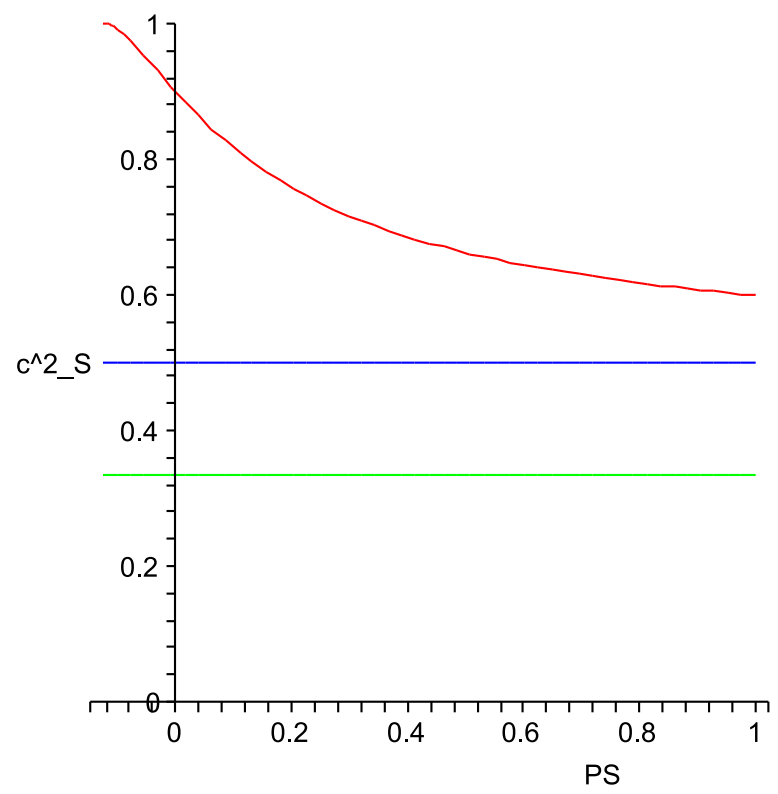

Figure 4. The speed of sound for an electrically neutral, extremal, black hole (with $c=1$ ).

The square of the speed of sound for an extremal electrically neutral black hole is plotted in figure 4 , for $P S>-1 / 8$. For comparison the asymptotic value $\left(c_{S}^{2}=1 / 2\right.$ for $\left.P S \rightarrow \infty\right)$ and the speed of sound in a thermal gas of photons $\left(c_{\gamma}^{2}=1 / 3\right)$ are also shown.

\section{Conclusions}

In conclusion there are strong reasons to believe that the cosmological constant should be included in the laws of black hole thermodynamics as a thermodynamic variable, proportional to the pressure of ordinary thermodynamics. The conjugate variable is a thermodynamic volume (10) and the complete first law of black hole thermodynamics is now (8), 


$$
d U=T d S+\Omega d J+\Phi d Q-P d V
$$

With this interpretation the ADM mass of the black hole is identified with the enthalpy

$$
M=H(S, P, J, Q)=U(S, V, J, Q)+P V
$$

rather than the internal energy, $U$, of the system.

The inclusion of this extra term increases the maximal efficiency of a Penrose process: for a neutral black hole in asymptotically anti-de Sitter space the maximal efficiency is increased from 0.2929 in asymptotically flat space to 0.5184 in the asymptotically AdS case. For a charged black hole the efficiency can be as high as $75 \%$. A positive cosmological constant is expected to reduce the efficiency of a Penrose process below the asymptotically flat space value.

This point of view makes the relation between asymptotically AdS black holes and the Van der Waals gas, first found in [35, 36], even closer as there is now a critical volume associated with the critical point. The thermodynamic volume then plays the rôle of an order parameter for this phase transition and the critical exponents take the mean field values,

$$
\alpha=0, \quad \beta=\frac{1}{2}, \quad \gamma=1, \quad \delta=3
$$

While there is no second order phase transition for a black hole in de Sitter space, there are other possible physical effects of including the $P d V$ term in the first law. The adiabatic compressibility can be calculated (93) and the speed of sound for such a black hole (95) is greater even than that of a photon gas and approaches $c$ when $P S=-1 / 8$.

Despite much progress the thermodynamics of black holes in de Sitter space-time is still very poorly understood and no doubt much still remains to be discovered.

\section{Author details}

Brian P. Dolan ${ }^{1,2}$

1 Department of Mathematical Physics, National University of Ireland Maynooth, Maynooth, Co. Kildare, Ireland

2 School of Theoretical Physics, Dublin Institute for Advanced Studies, Dublin, Ireland

\section{References}

[1] S W Hawking. Black hole explosions? Nature, 248:30, 1974.

[2] S W Hawking. Particle creation by black holes. Comm. Math. Phys., 43:199, 1975. Erratum: ibid. vol. 46, p. 206 (1976). 
[3] S W Hawking. Black holes and thermodynamics. Phys. Rev., D13:191, 1976.

[4] R. Penrose. Gravitational collapse: the role of general relativity. Riv. Nuovo Cimento, 1:252, 1969.

[5] D. Christodoulou. Reversible and irreversible transformations in black-hole physics. Phys. Rev. Lett., 25:1596, 1970.

[6] D. Christodoulou and R. Ruffini. Reversible transformations of a charged black hole. Phys. Rev., D4:3552, 1971.

[7] J D Bekenstein. Title. Lett. Nuovo. Cimento, 4:737, 1972.

[8] J D Bekenstein. Black holes and entropy. Phys. Rev., D7:2333, 1973.

[9] R M Wald. General Relativity. University of Chicago Press, 1984.

[10] A G Riess et al. Observational evidence from supernovae for an accelerating universe and a cosmological constant. Astronomical Journal, 116:1009, 1998.

[11] S. Perlmutter et al. Measurements of $\Omega$ and $\Lambda$ from 42 high-redshift supernovae. Astrophysical Journal, 517:565, 1999. [arXiv:astro-ph/9812133].

[12] B. Lukács and K. Martinás. Thermodynamics of negative absolute pressures. Acta. Phys. Pol., B21:177, 1990.

[13] M. Henneaux and C. Teitelboim. The cosmological constant as a canonical variable. Phys. Lett., 143B:415, 1984.

[14] M. Henneaux and C. Teitelboim. The cosmological constant and general covariance. Phys. Lett., 222B:195, 1989.

[15] M. Henneaux and C. Teitelboim. Asymptotically anti-de sitter spaces. Commun. Math. Phys., 98:391, 1985.

[16] C. Teitelboim. The cosmological constant as a thermodynamic black hole parameter. Phys. Lett., 158B:293, 198.

[17] Y. Sekiwa. Thermodynamics of de Sitter Black Holes: thermal cosmological constant. Phys. Rev., D73:084009, 2006. [arXiv:hep-th/0602269].

[18] E A Larrañaga Rubio. Stringy generalization of the first law of thermodynamics for rotating BTZ black hole with a cosmological constant as state parameter. [arXiv:0711.0012 [gr-qc]], 2007.

[19] S. Wang, S-Q. Wu, F. Xie, and L. Dan. The first laws of thermodynamics of the (2+1)-dimensional BTZ black holes and Kerr-de Sitter spacetimes. Chin. Phys. Lett., 23:1096, 2006. [arXiv:hep-th/0601147].

[20] S. Wang. Thermodynamics of high dimensional Schwarzschild de Sitter spacetimes: variable cosmological constant. [arXiv:gr-qc/0606109], 2006. 
[21] M. Urano, A. Tomimatsu, and H. Saida. Mechanical first law of black hole space- times with cosmological constant and its application to Schwarzschild-de Sitter spacetime. Class. Quantum Grav., 26:105010, 2009. arXiv:0903.4230 [gr-qc].

[22] S. Weinberg. The cosmological constant problem. Rev. Mod. Phys., 61:1, 1989.

[23] D. Kastor, S. Ray, and J. Traschen. Enthalpy and the mechanics of AdS black holes. Class. Quantum Grav., 26:195011, 2009. [arXiv:0904.2765 [hep-th]].

[24] L Smarr. Mass formula for Kerr black holes. Phys. Rev. Lett., 30:71, 1973. Erratum: ibid. vol. 30, p. 521 (1973).

[25] M.K. Parikh. Volume of black holes. Phys. Rev., D73:124021, 2006.

[26] W. Ballik and K. Lake. The volume of stationary black holes and the meaning of the surface gravity. 2012. [arXiv:1005.1116 [gr-qc]].

[27] M M Caldarelli, G. Cognola, and D. Klemm. Thermodynamics of Kerr-Newman-AdS black holes and conformal field theories. Class. Quantum Grav., 17:399, 2000. [arXiv:hep-th/9908022].

[28] B P Dolan. The cosmological constant and black hole thermodynamic potentials. Class. Quantum Grav., 28:125020, 2011. [arXiv:1008.5023].

[29] B. Carter. Hamiltonian-Jacobi and Schrödinger separable solutions of Einstein's equations. Comm. Math. Phys., 10:280, 1968.

[30] B P Dolan. Pressure and volume in the first law of black hole thermodynamics. Class. Quantum Grav., 28:235017, 2011. [arXiv:1106.6260].

[31] M. Cvetic, G W Gibbons, D. Kubiznák, and C N Pope. Black hole enthalpy and an entropy inequality for the thermodynamic volume. Phys. Rev., D84:024037, 2011. [arXiv:1012.2888[hep-th].

[32] A. Maloney and E. Witten. Quantum gravity partition functions in three dimensions. JHEP, 1002:029, 2010. [arXiv:0712.0155 [hep-th]].

[33] M Bañadis, C Teitelboim, and J Zanelli. The black hole in three dimensional space time. Phys. Rev. Lett., 69:1849, 1992.

[34] S W Hawking and D N Page. Thermodynamics of black holes in anti-de Sitter space. Comm. Math. Phys., 87:577, 1983.

[35] A Chamblin, R Emparan, C V Johnson, and R C Myers. Charged AdS black holes and catastrophic holography. Phys. Rev., D60:064018, 1999. [arXiv:hep-th/9902170v2].

[36] A Chamblin, R Emparan, C V Johnson, and R C Myers. Holography, thermodynamics and fluctuations of charged AdS black holes. Phys. Rev., D60:104026, 1999. [arXiv:hep-th/9904197]. 
[37] D. Kubiznak and R B Mann. P-V criticality of charged AdS black holes. arXiv:[1205.0559], 2012.

[38] M Sewell. On legendre transformations and elementary catastrophes. Math. Proc. Camb. Phil. Soc., 82:147, 1977.

[39] L D Landau and E M Lifshitz. Statistical Physics, volume 5 of A Course of Theoretical Physics. Pergammon, Oxford, Part 1, 3rd edition, 1980.

[40] N. Goldenfeld. Lectures On Phase Transitions And The Renormalization Group (Frontiers in Physics;85). Addison-Wesley, 1992.

[41] V I Arnold. Catastrophe Theory. Springer-Verlag, 1984.

[42] B P Dolan. Compressibility of rotating black holes. Phys. Rev., D84:127503, 2011. [arXiv:1109.0198].

[43] O Aharony, S S Gubser, J Maldacena, H Ooguri, and Y Oz. Large N field theories, string theory and gravity. Phys. Rep., 323:183, 2000. [arXiv:hep-th/9905111].

[44] S A Hartnoll. Lectures on holographic methods for condensed matter physics. Class. Quant. Grav., 26:224002, 2009. [arXiv:0903.3246v3 [hep-th]].

[45] A Gomberoff and C Teitelboim. de Sitter black holes with either of the two horizons as a boundary. Phys. Rev., D67:104024, 2003. [arXiv:hep-th/0302204].

[46] $\mathrm{T}$ Roy Choudhury and $\mathrm{T}$ Padmanabhan. Concept of temperature in multi-horizon spacetimes: Analysis of Schwarzschild-de Sitter metric. Gen. Rel. Grav., 39:1789, 2007. [arXiv:gr-qc/0404091].

[47] A Corichi and A Gomberoff. Black holes in de Sitter space: Masses, energies and entropy bounds. Phys. Rev., D69:064016, 2004. [arXiv:hep-th/0311030].

[48] R Aros. de Sitter thermodynamics: A glimpse into non equilibrium. Phys. Rev., D77:104013, 2008. [arXiv:0801.4591].

[49] B J Carr. Primordial black holes as a probe of cosmology and high energy physics. Lect. Notes Phys., 631:301, 2003. [arXiv:astro-ph/0310838].

[50] S. Gunasekaran, D. Kubizn̆ák and R.B. Mann, "Extended phase space thermodynamics for charged and rotating black holes and Born-Infeld vacuum polarization, [arXiv:1208.6251] 
